\title{
A Novel Method for the Induction of Experimental Glaucoma Using Magnetic Microspheres
}

\author{
Paulina A. Samsel, ${ }^{1}$ Lilian Kisiswa, ${ }^{1}$ Jonatban T. Erichsen, ${ }^{1}$ Stepben D. Cross, ${ }^{1}$ \\ and James E. Morgan ${ }^{1,2}$
}

Purpose. The development of a method for the sustained elevation of intraocular pressure in experimental glaucoma based on the anterior chamber injection of paramagnetic microbeads.

MethoDs. Unilateral glaucoma was induced in adult male Norwegian Brown rats by the injection of paramagnetic polystyrene microspheres. A handheld 0.45 Tesla magnet was used to draw the beads into the iridocorneal angle to impede aqueous drainage via the trabecular meshwork.

Results. Elevated intraocular pressures (IOPs) were induced in 61 rats, resulting in a mean elevation of $5.8 \mathrm{~mm} \mathrm{Hg} \pm 1.0$ (SEM) relative to the contralateral control eye. The mean duration of sustained IOP elevation (defined as $>5 \mathrm{~mm} \mathrm{Hg}$ relative to the control eye for at least 7 consecutive days) after a single injection was 12.8 days \pm 0.9 (SEM, maximum duration 27 days). In all eyes, the visual axis remained clear from the time of injection, with minimal inflammation after injection. Retinal ganglion cell loss was determined in 21 animals (mean integral IOP, $194.5 \mathrm{~mm} \mathrm{Hg}$ days $\pm 87.5[\mathrm{SEM}])$ as $36.4 \% \pm 2.4$ (SEM) compared with the contralateral, untreated eye.

Conclusions. The use of paramagnetic microbeads for the occlusion of the iridocorneal angle produces a sustained elevation of IOP with fewer injections and avoids the risk of visual axis occlusion. It represents a simple and effective method for the induction of experimental glaucoma. (Invest Ophthalmol Vis Sci. 2011;52:1671-1675) DOI:10.1167/iovs.09-3921

$\mathrm{C}$ onsiderable advances have been made in our understanding of the pathophysiology of glaucoma, helped in part by the availability of cost-effective models of disease based on rodents, such as the rat and mouse. ${ }^{1,2}$ Although spontaneous genetic models of glaucoma exist, ${ }^{3}$ the use of models in which the precise onset of glaucoma can be determined is important for grading diseases and the evaluation of novel therapies.

A variety of methods have been developed for the elevation of intraocular pressure (IOP), each with advantages and disadvantages. For example, cautery of episcleral vessels, ${ }^{4-7}$ perhaps the simplest method, runs the risk that it might create venous drainage of the choroid and therefore induce retinal effects unrelated to the elevation of IOP. Other methods, such as episcleral vein sclerosis with hypertonic saline $\mathrm{IOP}^{8-10}$, limit the outflow of aqueous at the level of the trabecular meshwork

From the Schools of ${ }^{1}$ Optometry and Vision Sciences and ${ }^{2}$ Medicine, Cardiff University, Cardiff, United Kingdom.

Supported by the National Eye Research Centre UK Grant No. SCIAD050.

Submitted for publication April 27, 2009; revised January 6, March 19, and July 23, 2010; accepted August 2, 2010

Disclosure: P.A. Samsel, None; L. Kisiswa, None; J.T. Erichsen, None; S.D. Cross, None; J.E. Morgan, Pfizer (F)

Corresponding author: James E. Morgan, School of Optometry and Vision Sciences, Cardiff University, Maindy Road, Cardiff CF24 4LU, UK; morganje3@cardiff.ac.uk. to produce stable and moderate elevations of IOP with a low risk of choroidal damage. ${ }^{11}$ However, the injections can be technically challenging. As a result of these considerations, alternative methods have been sought to induce experimental glaucoma in rodents. Laser occlusion of the episcleral vessels ${ }^{12-16}$ can be effective but works best with albino animals and requires use of a laser facility. More recently, methods based on obstruction of the trabecular meshwork with microscopic beads have been used, ${ }^{7,17}$ after successful application of this technique in primates. ${ }^{18}$ The injection of microbeads into the anterior chamber is straightforward, does not require specialist equipment, and can be applied to a range of species with only minor modification. Furthermore, the IOP elevation can be modulated with subsequent injections. The principal disadvantage is that beads can be difficult to retain after injection, resulting in a variable increase in IOP. The location of the beads within the anterior chamber can also be difficult to control; if beads locate to the pupillary zone, they can compromise fundoscopic monitoring of optic nerve damage.

To address these issues, we have developed a novel technique for the induction of ocular hypertension using paramagnetic microbeads. The beads have the advantage that they can be directed to the iridocorneal angle in the rodent eye to optimize occlusion of the trabecular meshwork and facilitate visualization of the fundus. Subsequent injections can be given, as required, to further modulate the elevation in IOP.

\section{MAterials AND Methods}

\section{Induction of Elevated IOP}

All experimental procedures complied with Home Office (UK) regulations and the ARVO Statement for the Use of Animals in Ophthalmic and Vision Research. Sixty-one retired male breeder Brown Norway rats were housed in a constant low-light environment (40-60 lux) to minimize diurnal fluctuations in IOP, with food and water provided ad libitum. IOPs were measured three times before injection and daily or every 2 to 3 days after injection using a rebound tonometer (TonoLab; Tiolat, Oy, Finland) calibrated for use with the rat eye. ${ }^{19}$ All measurements were made in awake animals in which the cornea was anesthetized using topical $0.4 \%$ oxybuprocaine hydrochloride eye drops (Chauvin, Kingston-Upon-Thames, UK). The IOP was taken as the mean and SD of five readings.

In each animal, the IOP was elevated in the left eye by injecting a sterile balanced salt solution (BSS; Alcon UK, Hemel Hempstead, UK) containing $30 \mathrm{mg} / \mathrm{mL}$ ferro-magnetic microspheres (Corpuscular Inc, Cold Spring, NY; bead diameter, $5 \mu \mathrm{m}$ ) into the anterior chamber of the left eye. Microspheres were sterilized by $\gamma$-irradiation (Gammacell 1000 Elite Caesium Source, 22 TBq; Nordon International, Inc., Ontario, Canada). All injections were made under isoflurane anesthesia, with topical chloramphenicol $(0.5 \%)$ administered pre- and post-injection (Chauvin). Approximately $10-20 \mu \mathrm{L}$ was injected into the anterior chamber of the left eye, delivering approximately $0.3-0.6 \mathrm{mg}$ of beads. The right eye acted as an unoperated control. Injections were made 


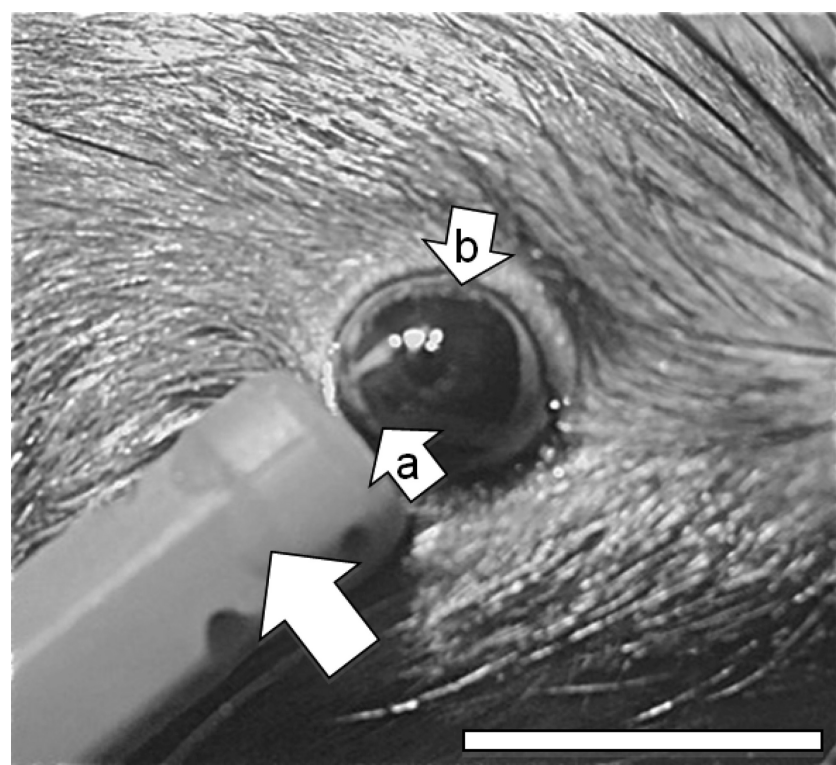

FIGURE 1. Magnet (large arrow) used to direct beads to the anterior chamber angle. The smaller arrows show the location of beads in the iridocorneal angle. Arrow $a$ indicates beads adjacent to the magnet that are being drawn into the angle. Arrow $b$ shows the location of beads contralateral to the magnet that were previously drawn into the angle and remain in situ. Scale bar, $1 \mathrm{~cm}$.

using a 32- or 33-gauge needle (Hamilton Corporation, Bonaduz, Switzerland; and WPI Ltd, Stevenage, UK, respectively) inserted parallel to the iris. The 33-gauge needles were beveled on three sides to facilitate injection through the cornea and provided the easiest transit through the corneal stroma. A tunneled injection was made with the needle running parallel to the anterior surface of the iris to minimize the risk of iris trauma. Since the beads tended to settle in the syringe under the influence of gravity, the syringe and needle were agitated using a vortex stirrer immediately before injection to resuspend the beads in BSS. The minimum period between subsequent injections was 1 week.

Once the injection of beads was completed, the needle was kept in position to ensure maintenance of anterior chamber depth, and the beads then drawn away from the injection site using a small handheld magnet $(0.45$ Tesla), thereby minimizing the egress of beads from the injection track. The magnet was then used to distribute the microspheres around the iridocorneal angle to reduce the outflow of aqueous humor via the trabecular meshwork. Immediate increases in IOP could be moderated by allowing the leakage of aqueous around the injection cannula as it was withdrawn from the eye. Attempts to do this without redirection of beads resulted in the loss of beads via the injection site. Microsphere injections were performed up to three times in the left eye, depending on the required level and duration of the IOP elevation. For the purposes of control studies, five rats were injected with $20 \mu \mathrm{L}$ of magnetic microspheres without magnetic direction to the iridocorneal angle. Five rats received a vehicle injection of $20 \mu \mathrm{L}$ BSS (no beads) into the anterior chamber.

All rats were subsequently killed by an overdose of $\mathrm{CO}_{2}$ and selected retinas prepared as whole mounts for the quantification of cell loss in the retinal ganglion cell (RGC) layer. Anterior segment images of the beads distribution were taken as required with a camera (Nikon Coolpix 4500; Nikon, Tokyo, Japan) attached to a slitlamp (Haag Streit UK Ltd, Harlow, UK). Cryosections $(10 \mu \mathrm{m})$ of the anterior chambers prepared on gelatin-coated slides were stained with hematoxylin and eosin for bright-field microscopic imaging or mounted under DPX (Raymond A. Lamb) for DIC imaging. Section images were taken using a bright-field microscope (Leica DMRA2; Leica Microsystems [UK] Ltd, Milton Keynes, UK) and associated software (Leica QWin v.3; Leica).

\section{Neuron Nuclei Imaging}

For the quantification of RGC loss, retinal whole mounts were stained (Hoechst 33342; Sigma-Aldrich, Gillingham, UK) and mounted in anti-
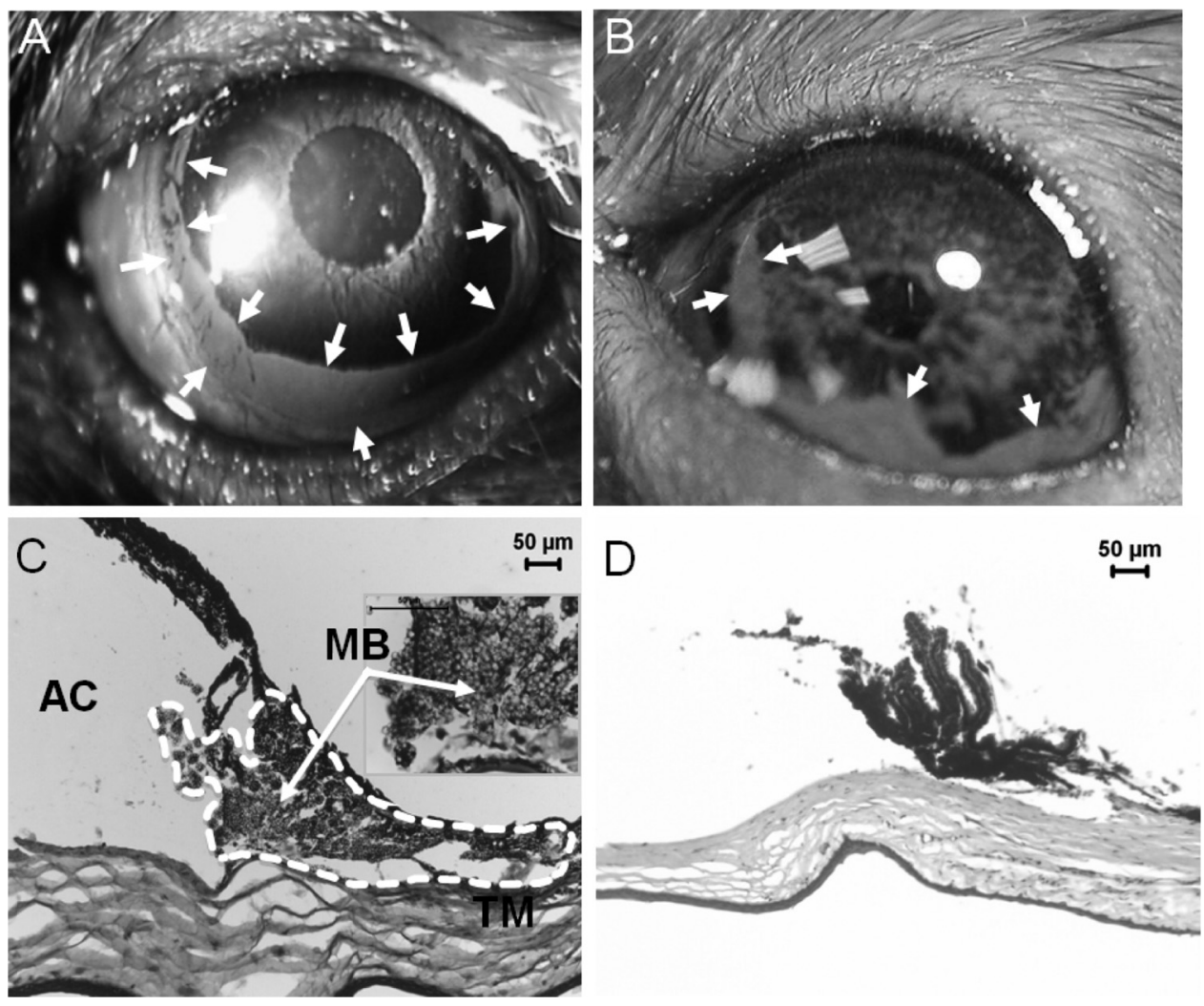

Figure 2. (A) Anterior segment image showing the distribution of paramagnetic beads immediately after magnetic redirection. Arrows indicate the distribution of beads in the iridocorneal angle. After magnetic direction, the beads become compacted into the iridocorneal angle and are therefore discernible as a band rather than as individual beads. (B) Anterior segment image showing the distribution of paramagnetic beads without using a magnet immediately post-injection. $A r$ rows indicate beads in the anterior chamber angle and the iris surface, extending to the pupil. (C) Cryosection of the anterior chamber injected with magnetic beads showing complete occlusion of the iridocorneal angle. The eye was enucleated 32 days post-injection. The mean IOP increase for this eye was $19.15 \mathrm{~mm} \mathrm{Hg}$. The integral IOP increase was $470.83 \mathrm{~mm} \mathrm{Hg}$ days. $\mathrm{AC}$, anterior chamber; $\mathrm{MB}$, microbeads; TM, trabecular meshwork. Inset image shows higher magnification image of the angle. The bead distribution is demarcated by the dashed line. (D) Cryosection of the anterior chamber from the control eye. 
fade medium (ProLong Gold; Invitrogen Ltd, Paisley, UK). The number of cells in the RGC layer was counted to provide an index of cell damage. Although amacrine cells would have been included in the analysis, ${ }^{20,21}$ this has been validated in the mouse glaucoma model as a useful measure of RGC damage that avoids the variability associated with RGC labeling. ${ }^{22}$

Images of the RGC layer were obtained using a fluorescent microscope (Leica 6000B; Leica) linked to software (Stereo Investigator; MicroBrightField, Magdeburg, Germany). Retinal specimens were placed on a motorized stage, and the cells in the retinal ganglion cell layer (RGCL) were imaged with a $\times 20$ objective in 12 fields (sample area, 11,3095 $\mu \mathrm{m}^{2}$ ) arranged in a grid centered on the optic nerve. The number of labeled cells was counted manually (Cell Counter plug-in for ImageJ; NIH Images, NIH, Bethesda, MD). Approximately $2.4 \%$ of the total retinal area was sampled to estimate neuronal loss when compared with the contralateral control eye. Standard morphologic criteria were applied for discriminating nonneuronal cells (endothelial and glial cells) from neuronal cells.

\section{Statistics}

Statistical analysis was performed using standard statistical software (SPSS v.16.0 for Windows; SPSS, Chicago, IL). Normally distributed data for the peak and integral IOP for the eyes with and without hemorrhages were compared using independent-sample $t$-tests. IOP measurements for single animals were expressed as mean \pm SD (SD). Unless stated otherwise, group values were expressed as means and standard errors of the mean (SEM). Pearson's correlation coefficient was used to test the correlation between peak IOP increase and cell loss and mean IOP elevation and cell loss. The RGC layer counts in control and experimental groups were compared using the independent-sample $t$-test. The degree of cell loss in different retinal regions was compared using a one-way ANOVA. Normally distributed data for the mean IOP of the injected and contralateral eyes were compared using independent-sample $t$-test.

\section{RESUlTS}

\section{IOP Elevation}

Microbead injections were made in 61 rats. Figure 1 demonstrates the technique for directing the microspheres to the iridocorneal angle with the handheld external magnet. The magnet strength was selected to exert local control of the microbead distribution; beads lodged in the iridocorneal angle opposite the magnet were not displaced. The typical bead distribution after injection and redirection with the external magnet is shown in Figure 2A. With sufficient anterior chamber depth, the beads could easily be directed to provide uniform occlusion of the iridocorneal angle, a process facilitated by retention of the cannula in the anterior chamber during bead redistribution. Histologic examination of the anterior segment of the eye after a single intraocular bead injection confirmed sequestration of the beads within the trabecular meshwork (Fig. 2C) in contrast with an uninjected contralateral eye (Fig. 2D).

Microbead injections were well tolerated and did not incite a uveitic response. Localized iris hemorrhages were noted if the tip of the injection cannula damaged superficial vessels, but these were small and resolved within a few days. The IOP profiles after injection could be classified either as a sustained increase in IOP (Fig. 3A; 64\% of eyes) or a fluctuating increase (Fig. 3B; 36\% of eyes). A sustained IOP increase (defined as an elevation in IOP $5 \mathrm{~mm} \mathrm{Hg}$ greater than the contralateral eye on at least 7 consecutive days) was obtained in $64 \%$ of rats. The mean duration for the IOP increase after a single injection was $12.8 \pm 0.9$ days with a maximum duration of 27 days. Since a 7-to-14-day period was allowed to elapse after the fall in IOP (to confirm that the fall in IOP was persistent) the mean time
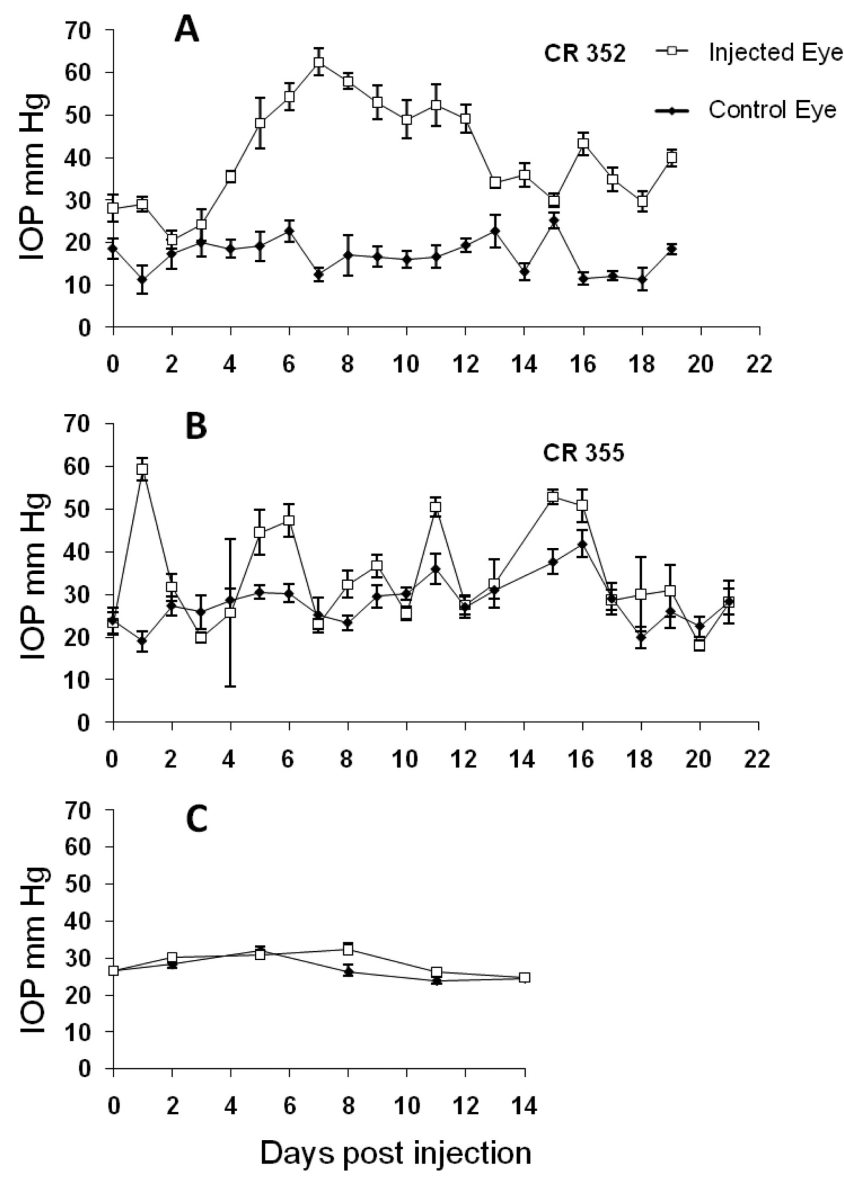

Figure 3. IOPs after single bead injections followed by using a magnet to direct beads to the anterior chamber angle in rats CR352 (A) and CR355 (B). IOPs after a single bead injection without using a magnet in rat CR461 showing the transient increase in IOP (C). Error bars, SD; open symbols, injected eye; closed symbols, control eye.

between injections (26.9 days \pm 5.4) exceeded the mean duration of IOP increase. Up to three injections were made in any single animal. Fifty-one animals received injections with 20 $\mu \mathrm{L}$, four with $10 \mu \mathrm{L}$, and six with $15 \mu \mathrm{L}$ Injections were repeated to prevent the IOP falling to within $5 \mathrm{~mm} \mathrm{Hg}$ of the contralateral control eye.

The mean IOP for all the animals was $29.4 \pm 0.9 \mathrm{~mm} \mathrm{Hg}$ for injected eyes and $23.6 \pm 0.4 \mathrm{~mm} \mathrm{Hg}$ in the contralateral untreated eyes $(P<0.001)$. The mean and peak IOP increases were $5.8 \pm 1.0$ and $35.7 \pm 1.0 \mathrm{~mm} \mathrm{Hg}$, respectively, with a mean integral IOP increase of $118.0 \pm 57.4 \mathrm{~mm} \mathrm{Hg}$ days. Animals were monitored for a mean interval for $74 \pm 5.4$ days (range 11 to 178 days) during which they were regularly checked for signs of inflammation. The injection of the beads did not result in any long-term inflammatory response.

Figure 4 shows the mean intraocular pressure (IOP) in experimental and contralateral eyes in all experimental rats during the first 42 days after injection. Eyes injected with $10 \mu \mathrm{L}$ $(n=12)$ or $20 \mu \mathrm{L}$ of beads $(n=51)$ did not demonstrate a significant difference in either the mean IOP increase $(6.0 \pm$ 1.5 vs. $6.2 \pm 1.2 \mathrm{~mm} \mathrm{Hg}$ ) or peak IOP $(31.05 \pm 2.0 \mathrm{~mm} \mathrm{Hg}$ vs. $35.8 \pm 1.2 \mathrm{~mm} \mathrm{Hg}$ ).

To monitor the effect of bead injections alone, beads were injected $(20 \mu \mathrm{L}$, five animals $)$ at the same concentration but not manipulated under the control of a magnetic field. Aqueous was allowed to egress after the injection to ensure that the IOP was normalized. After injection, the majority of beads settled on the inferior part of the anterior chamber, near the injection 


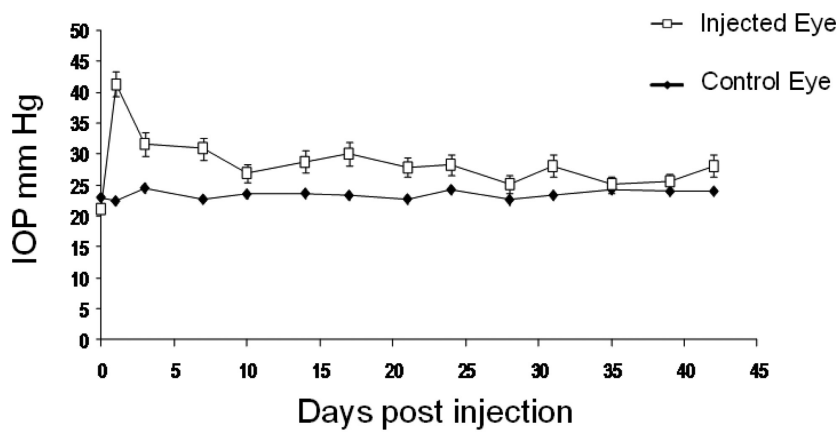

FIGURE 4. The mean IOP in all experimental rats during the first 42 days after injection. Error bars, SEM; open symbols, injected eyes; closed symbols, control eyes.

site (Fig. 2B). The remaining beads were spread around the anterior chamber. The IOP was measured every third day and the animals monitored for 2 weeks post-injection. Figure 3C shows the mean IOP profile injected rats in this group. The IOP in the bead group did not show a significant increase (mean IOP 28.71 [4.88] injected eye versus 27.04 [4.07] control eye; $P=0.21)$. In a further five animals vehicle injections $(20 \mu \mathrm{L}$ BSS) were made unilaterally with normalization of the IOP post-injection. We observed a slight increase in IOPs in the injected eyes over the 2-week period (mean IOP 30.03 [3.20] injected eye versus 26.06 [2.91] control eye; $P=0.002$ ).

\section{Neuron Quantification in the RGCL}

All retinas were dissected out and prepared as whole mount preparations. We observed discrete $(<100 \mu \mathrm{m})$ retinal hemorrhages in a peripapillary distribution in 5 of the 61 eyes. RGCL neuronal populations were sampled in 21 animals with mean IOP elevation $7.7 \pm 1.4 \mathrm{~mm} \mathrm{Hg}$ and mean integral IOP $194.5 \pm$ $87.5 \mathrm{~mm} \mathrm{Hg}$ days. The mean density of cells in the RGC layer in control retinas was $4080 \pm 99$ cells $/ \mathrm{mm}^{2}$ and $2593 \pm 139$ cells $/ \mathrm{mm}^{2}$ in glaucomatous retinas, accounting for $36.4 \pm 2.4 \%$ cell loss $(P=0.002)$. In a linear regression analysis we did not observe a significant relationship between the degree of cell loss relative to the contralateral control eye for the duration of IOP increase $\left(r^{2}=0.18, P=0.56\right)$, peak or mean IOP increase, or area under the IOP curve. We did not observe a consistent difference in retinal regions when samples were taken at 1,2 , or $3 \mathrm{~mm}$ from the optic disc for the degree of loss $(P=0.98$, one-way ANOVA; see Table 1). RGC quantification in the bead (no magnetic manipulation) and BSS injection animals did not show any significant difference from controls.

\section{Discussion}

We describe a simple and effective method for the elevation of IOP in the rat. The technique is notable in that it is technically undemanding and provides a measure of control over the distribution of beads within the anterior segment. The ability to draw the beads away from the visual axis and to distribute them around the iridocorneal angle cleared the visual axis to facilitate examination of the retina and optic disc.

The principal finding of the present study is that fewer injections may be required to achieve a sustained increase in IOP compared with injections using nonmagnetic beads. Thus, the injection of latex microspheres, in combination with hydroxypropylmethylcellulose or when given alone required six to nine weekly injections, respectively, to achieve sustained elevation in IOP. ${ }^{7}$ Similarly, eight to 10 injections were necessary to produce a sustained IOP elevation that lasted up to 2 weeks in the primate ${ }^{18}$; for more prolonged IOP elevation in the primate, as many as 20 bead injections were required to maintain significant occlusion of the iridocorneal angle. In the present study we achieved a sustained IOP increase in $64 \%$ of animals after up to three injections, lasting on average $12.8 \pm$ 0.9 days. The mean IOP in injected eyes in our model, $29.4 \pm$ $0.9 \mathrm{~mm} \mathrm{Hg}$, was similar to the mean IOP seen eyes injected with latex microspheres $(28.1 \pm 0.7 \mathrm{~mm} \mathrm{Hg}){ }^{7}$ We were interested to note that the increase in IOP was not consistently related to the volume of the bead injection. One likely explanation is that the egress of aqueous seen after removal of the needle tended to normalize the IOP regardless of the injection volume. The present study used beads of approximately $5 \mu \mathrm{m}$ diameter, which was smaller than the $10 \mu \mathrm{m}$ beads used in previous studies. ${ }^{7,18}$ Recently the use of $15 \mu \mathrm{m}$ beads has been reported to achieve sustained IOP elevations of up to 8 weeks based on two injections in rats, suggesting that the use of larger beads may be important in the long-term induction of ocular hypertension. ${ }^{17}$ The bead diameter of $5 \mu \mathrm{m}$ was selected because this seemed most likely to occlude the spaces reported in the rodent trabecular meshwork. ${ }^{23}$ In preliminary experiments, the use of beads in the 1-2 $\mu \mathrm{m}$ range did not produce a consistent increase in IOP and had the disadvantage that the beads were more difficult to guide in the anterior chamber. A critical technical point is that we removed the carrier fluid in which the beads are packaged to minimize the introduction of potentially toxic agents into the anterior chamber.

For animals in which beads were not redirected, we did not observe a significant, sustained elevation in IOP. Even in the animals injected with $20 \mu \mathrm{L}$ of beads we did not observe a significant reduction in RGC layer cell density compared with animals with a sustained elevation in IOP. Taken together, these observations suggest that the loss of cells in the RGC layer results from the effects of sustained IOP increase, rather than from any transitory increase in IOP as a result of injection. The lack of RGC layer cell loss in the animal injected with BSS alone is consistent with this conclusion.

The degree of cell loss in our model is similar to that reported for other models. The use of (nonmagnetic) microbead occlusion in a rat glaucoma model ${ }^{7}$ produced a level RGC loss $(23.1 \pm 2.1 \%)$ similar to that seen in the present study $(36.4 \pm 2.4 \%)$. Comparable RGC loss $(30 \%-40 \%)$ has also been reported with the hypertonic saline model after moderate elevation in $\mathrm{IOP}^{24,25}$ and the episcleral vein cautery model

TABLE 1. Cell Density, Mean Number of Cells per Retina, and Cell Loss in the RGCL for Control and Glaucomatous Retinas

\begin{tabular}{|c|c|c|c|c|c|}
\hline \multirow[b]{2}{*}{ Eccentricity } & \multicolumn{2}{|c|}{ Control Retinas } & \multicolumn{2}{|c|}{ Glaucomatous Retinas } & \multirow[b]{2}{*}{$\begin{array}{l}\text { Percentage Cell Loss } \\
\quad(\text { mean } \pm \text { SEM })\end{array}$} \\
\hline & $\begin{array}{c}\text { Cell Density } \\
\left(\text { cell } / \mathbf{m m}^{2} \pm \text { SEM }\right)\end{array}$ & $\begin{array}{l}\text { Cell Count } \\
(n \pm \text { SEM })\end{array}$ & $\begin{array}{c}\text { Cell Density } \\
\left(\text { cell } / \mathbf{m m}^{2} \pm \text { SEM }\right)\end{array}$ & $\begin{array}{l}\text { Cell Count } \\
(n \pm \text { SEM })\end{array}$ & \\
\hline $1 \mathrm{~mm}$ & $4170 \pm 104$ & $471 \pm 12$ & $2701 \pm 14$ & $305 \pm 14$ & $35.2 \pm 2.5$ \\
\hline $2 \mathrm{~mm}$ & $4327 \pm 98$ & $489 \pm 11$ & $2699 \pm 144$ & $305 \pm 16$ & $37.6 \pm 2.3$ \\
\hline $3 \mathrm{~mm}$ & $3745 \pm 96$ & $423 \pm 11$ & $2381 \pm 145$ & $269 \pm 16$ & $36.4 \pm 2.6$ \\
\hline Mean & $4080 \pm 99$ & $461 \pm 11$ & $2593 \pm 139$ & $293 \pm 16$ & $36.4 \pm 2.4$ \\
\hline
\end{tabular}


$(30 \%-40 \%){ }^{5,26,27}$ We did not observe a consistent variation in the degree of cell loss as a function of retinal eccentricity in the sampled range (1-3 mm). By contrast, clustered RGC loss has been reported in the rat vein cautery model. ${ }^{28}$ Other studies have reported greater cell loss in the peripheral compared with central regions, but in animals with longer durations of IOP; ${ }^{4}$ this pattern of cell loss has been observed in a comparative study of bead injection, cautery, and hypertonic saline models, with the greatest effect seen with vein cautery. ${ }^{7}$

In summary, we present a simple method for the induction of experimental glaucoma in the rodent eye. It has the principal advantages over nonmagnetic microbead models in that the visual axis is not obscured after injection and that complete occlusion of the iridocorneal angle can be achieved with a single injection. We anticipate that it could be adapted with little modification for use in other species.

\section{References}

1. Morrison JC, Johnson E, Cepurna WO. Rat models for glaucoma research. Prog Brain Res. 2008;173:285-301.

2. John SW. Mechanistic insights into glaucoma provided by experimental genetics: the Cogan Lecture. Invest Ophthalmol Vis Sci. 2005;46:2649-2661.

3. John SW, Anderson MG, Smith RS. Mouse genetics: a tool to help unlock the mechanisms of glaucoma. J Glaucoma. 1999;8:400412 .

4. Laquis S, Chaudhary P, Sharma SC. The patterns of retinal ganglion cell death in hypertensive eyes. Brain Res. 1998;784:100-104.

5. Naskar R, Wissing M, Thanos S. Detection of early neuron degeneration and accompanying microglial responses in the retina of a rat model of glaucoma. Invest Ophthalmol Vis Sci. 2002;43:29622968.

6. Garcia-Valenzuela E, Shareef S, Walsh J, Sharma S. Programmed cell death of retinal ganglion cells during experimental glaucoma. Exp Eye Res. 1995;61:33-44.

7. Urcola JH, Hernandez M, Vecino E. Three experimental glaucoma models in rats: comparison of the effects of intraocular pressure elevation on retinal ganglion cell size and death. Exp Eye Res. 2006;83:429- 437 .

8. Nissirios N, Chanis R, Johnson E, et al. Comparison of anterior segment structures in two rat glaucoma models: an ultrasound biomicroscopic study. Invest Ophthalmol Vis Sci. 2008;49:24782482.

9. Morrison JC, Moore CG, Deppmeier LM, Gold BG, Meshul CK, Johnson EC. A rat model of chronic pressure-induced optic nerve damage. Exp Eye Res. 1997;64:85-96.

10. Morgan JE, Datta AV, Erichsen JT, Albon J, Boulton ME. Retinal ganglion cell remodelling in experimental glaucoma. Adv Exp Med Biol. 2006;572:397-402.

11. Morrison JC. Elevated intraocular pressure and optic nerve injury models in the rat. J Glaucoma. 2005;14:315-317.
12. Levkovitch-Verbin H, Quigley HA, Martin KR, Valenta D, Baumrind LA, Pease ME. Translimbal laser photocoagulation to the trabecular meshwork as a model of glaucoma in rats. Invest Ophthalmol Vis Sci. 2002; $43: 402-410$.

13. Martin KR, Levkovitch-Verbin H, Valenta D, Baumrind L, Pease ME, Quigley HA. Retinal glutamate transporter changes in experimental glaucoma and after optic nerve transection in the rat. Invest Ophthalmol Vis Sci. 2002;43:2236-2243.

14. Siu AW, Leung MC, To CH, Siu FK, Ji JZ, So KF. Total retinal nitric oxide production is increased in intraocular pressure-elevated rats. Exp Eye Res. 2002;75:401-406.

15. Schori H, Kipnis J, Yoles E, et al. Vaccination for protection of retinal ganglion cells against death from glutamate cytotoxicity and ocular hypertension: implications for glaucoma. Proc Natl Acad Sci U S A. 2001;98:3398-3403.

16. Chiu K, Chang R, So KF. Laser-induced chronic ocular hypertension model on SD rats. J Vis Exp. 2007;10:549.

17. Sappington RM, Carlson BJ, Crish SD, Calkins DJ. The microbead occlusion model: a paradigm for induced ocular hypertension in rats and mice. Invest Ophthalmol Vis Sci. 2010;51:207-216.

18. Weber AJ, Zelenak D. Experimental glaucoma in the primate induced by latex microspheres. J Neurosci Methods. 2001;111: $39-48$.

19. Wang WH, Millar JC, Pang IH, Wax MB, Clark AF. Noninvasive measurement of rodent intraocular pressure with a rebound tonometer. Invest Ophthalmol Vis Sci. 2005;46:4617-4621.

20. Cowey A, Perry VH. The projection of the temporal retina in rats, studied by retrograde transport of horseradish peroxidase. Exp Brain Res. 1979;35:457-464.

21. Perry V. Evidence for an amacrine cell system in the ganglion cell layer of the rat retina. Neuroscience. 1981;6:931-944.

22. Jakobs TC, Libby RT, Ben Y, John SW, Masland RH. Retinal ganglion cell degeneration is topological but not cell type specific in DBA/2J mice. J Cell Biol. 2005;171:313-325.

23. Smith RS, Zabaleta A, Savinova OV, John SW. The mouse anterior chamber angle and trabecular meshwork develop without cell death. BMC Dev Biol. 2001;1:3.

24. Hanninen VA, Pantcheva MB, Freeman EE, Poulin NR, Grosskreutz CL. Activation of caspase 9 in a rat model of experimental glaucoma. Curr Eye Res. 2002;25:389-395.

25. Fileta JB, Huang W, Kwon GP, et al. Efficient estimation of retinal ganglion cell number: a stereological approach. J Neurosci Methods. 2008;170:1-8.

26. Ahmed FA, Hegazy K, Chaudhary P, Sharma SC. Neuroprotective effect of alpha(2) agonist (brimonidine) on adult rat retinal ganglion cells after increased intraocular pressure. Brain Res. 2001; 913:133-139.

27. Neufeld AH, Sawada A, Becker B. Inhibition of nitric-oxide synthase 2 by aminoguanidine provides neuroprotection of retinal ganglion cells in a rat model of chronic glaucoma. Proc Natl Acad Sci U S A. 1999;96:9944-9948.

28. Mittag TW, Danias J, Pohorenec G, et al. Retinal damage after 3 to 4 months of elevated intraocular pressure in a rat glaucoma model. Invest Ophthalmol Vis Sci. 2000;41:3451-3459. 